\title{
summary
}

\section{Single tooth implants have acceptable 4-year survival rates}

Creugers NHJ, Kreulen CM, Snoek PA, deKanter RJAM. A systematic review of single-tooth restorations supported by implants. J. Dent 2000; 28:209-217

Objective To systematically review clinical studies on the performance of implant-supported single-tooth restorations (STR).

Data sources Medline 1990-1998, using keywords, 'dental in SB', 'implant' and 'single'.

Study selection Only English papers that met a strict three-level selection procedure, including availability of or the ability to calculate life-table survival curves, were included.

Data extraction and synthesis Cumulative life-table survival curves were constructed by pooling data from the included studies to new data sets.

Results Of 332 papers initially identified, the majority were excluded because they were case reports, technical notes or descriptions of clinical procedures. Nine studies met all the criteria with the data predicting a $97 \%( \pm 1 \%)$ survival implant survival at 4 years, with an uncomplicated crown survival rate of $83 \%( \pm 3 \%)$.

Conclusions Single tooth implants have an acceptable short-term survival rate. More information and better quality clinical studies and reporting are needed, however.

Address for reprints: Professor NHJ Creugers, Department of Occlusal Reconstruction and Oral Function, College of Dental Science, Faculty of Medical Sciences, University of Nijmegen 6500HB Nijmegen, The Netherlands. E-mail: n.creugers@dent.kun.nl

\section{Commentary}

The aim of this paper was a systematic review of clinical studies on the performance of implant-supported STR. The authors' assessment is correct that in studies of dental implants, failure/survival analyses should today include clinical performance criteria, for example, complications and failures of implant-supported restorations. These data should be collected in a format that allows life-table analysis.

In this review, the authors applied three selection levels to the original 332 papers identified from a MEDLINE search for inclusion in a combined analysis. After the first selection (criteria: STRs reported, clinical study, follow-up study), only 49 papers remained. Following the second selection (follow-up $=2$ years), 26 papers qualified, and after the third selection (sufficient data for life-table analysis of implants and/or STRs), 12 reports remained. Out of these, two out of two pairs of studies were dropped since they included the same implants. The homogeneity test for these remaining 10 papers revealed no differences in outcomes after 2 years of followup except for one study with significantly more STR complications. This study was excluded from the combined survival analysis.
The combined implant survival curve on 459 single-tooth implants in the nine remaining papers showed a survival rate of $97 \pm 1 \%$ after 4 years. This number is identical to that found in a recent meta-analyis on single-tooth implants after 7-8 years of follow-up. ${ }^{1}$

The combined curve for survival of the restorations showed a survival estimate of $83 \pm 3 \%$ after 4 years. It should be noted that this number is based on 240 STRs from four of the nine reports containing adequate information for a combined survival analysis.

An interesting and important point made by the authors in their discussion, related to the study that showed a significantly higher rate of STR complications and which was not, therefore, included in the combined analysis. When this study was included in a post-hoc data evaluation, it did not affect the implant survival rate $(97 \pm 1 \%)$, but reduced the STR survival rate to $79 \pm 3 \%$.

This should be a clear indication that in the process of evidence-based decision-making in dental treatment planning, the implant as an artificial root appears as a highly predictable alternative to a natural tooth root, and that the complications with implant therapy relate mostly to the suprastructure. As we recognise today, conventional fixed partial dentures used to replace a missing tooth show a survival of $75 \%$ after 15 years. $^{2}$ Problems with the abutment teeth (caries, periodontal disease, endodontic complication, root fracture, etc) are a major cause of failure of the supported restoration. Based on the information provided in the literature, this does not seem to apply to dental implants supporting STRs up to 8 years. ${ }^{1}$

Although the paper discussed here may have limited overall validity in terms of creating sufficient evidence for treatment decisionmaking, due to the limited numbers and the short observation time, it clearly makes a step in the right direction.

1. Lindh T, Gunne J, Tillberg A, Molin M. A meta-analysis of implants in partial edentulism. Clin Oral Implant Res 1998; 9:80-90.

2. Scurria MS, Bader JD, Shugars DA. Metaanalysis of fixed partial denture survival: prosthesis and abutments. J Prosthet Dent 1998; 79:495-564.

Hans-Peter Weber
Department of Restorative Dentistry,
Harvard School of Dental Medicine,
Boston, Massachusetts, USA

